\title{
Corrigendum: Candidate Gene Resequencing in a Large Bicuspid Aortic Valve-Associated Thoracic Aortic Aneurysm Cohort: SMAD6 as an Important Contributor
}

\section{OPEN ACCESS}

Edited and reviewed by: Gerald A. Meininger,

University of Missouri, United States

*Correspondence: Bart L. Loeys

bart.loeys@uantwerpen.be

${ }^{\dagger}$ These authors have contributed equally to this work.

Specialty section:

This article was submitted to

Vascular Physiology

a section of the journal

Frontiers in Physiology

Received: 09 August 2017 Accepted: 08 September 2017 Published: 25 September 2017

Citation:

Gillis E, Kumar AA, Luyckx I, Preuss C, Cannaerts $E$, van de Beek $G$,

Wieschendorf B, Alaerts M, Bolar N,

Vandeweyer G, Meester J,

Wünnemann F, Gould RA, Zhurayev $R$,

Zerbino D, Mohamed SA, Mital S,

Mertens L, Björck HM,

Franco-Cereceda A, McCallion AS,

Van Laer L, Verhagen JMA, van de Laar IMBH, Wessels MW, Messas E,

Goudot G, Nemcikova M, Krebsova A,

Kempers $M$, Salemink $S$,

Duijnhouwer $T$, Jeunemaitre $X$

Albuisson J, Eriksson P, Andelfinger G,

Dietz HC, Verstraeten A, Loeys BL and Mibava Leducq Consortium

(2017) Corrigendum: Candidate Gene

Resequencing in a Large Bicuspid

Aortic Valve-Associated Thoracic

Aortic Aneurysm Cohort: SMAD6 as an Important Contributor.

Front. Physiol. 8:730.

doi: 10.3389/fphys.2017.00730
Elisabeth Gillis ${ }^{1 \dagger}$, Ajay A. Kumar ${ }^{1 \dagger}$, Ilse Luyckx ${ }^{1}$, Christoph Preuss ${ }^{2}$, Elyssa Cannaerts ${ }^{1}$, Gerarda van de Beek ${ }^{1}$, Björn Wieschendorf ${ }^{1,3}$, Maaike Alaerts ${ }^{1}$, Nikhita Bolar ${ }^{1}$, Geert Vandeweyer ${ }^{1}$, Josephina Meester ${ }^{1}$, Florian Wünnemann ${ }^{2}$, Russell A. Gould ${ }^{4}$, Rustam Zhurayev ${ }^{5}$, Dmytro Zerbino ${ }^{5}$, Salah A. Mohamed ${ }^{3}$, Seema Mital ${ }^{6}$, Luc Mertens $^{6}$, Hanna M. Björck ${ }^{7}$, Anders Franco-Cereceda ${ }^{8}$, Andrew S. McCallion ${ }^{4}$, Lut Van Laer ${ }^{1}$, Judith M. A. Verhagen ${ }^{9}$, Ingrid M. B. H. van de Laar ${ }^{9}$, Marja W. Wessels ${ }^{9}$, Emmanuel Messas ${ }^{10}$, Guillaume Goudot ${ }^{10}$, Michaela Nemcikova ${ }^{11}$, Alice Krebsova ${ }^{12}$, Marlies Kempers ${ }^{13}$, Simone Salemink ${ }^{13}$, Toon Duijnhouwer ${ }^{13}$, Xavier Jeunemaitre ${ }^{10}$, Juliette Albuisson ${ }^{10}$, Per Eriksson ${ }^{7}$, Gregor Andelfinger ${ }^{2}$, Harry C. Dietz ${ }^{4,14}$, Aline Verstraeten ${ }^{1}$, Bart L. Loeys ${ }^{1,13^{*}}$ and Mibava Leducq Consortium

${ }^{1}$ Faculty of Medicine and Health Sciences, Center of Medical Genetics, University of Antwerp and Antwerp University Hospital, Antwerp, Belgium, ${ }^{2}$ Cardiovascular Genetics, Department of Pediatrics, CHU Sainte-Justine, Université de Montreal, Montreal, QC, Canada, ${ }^{3}$ Department of Cardiac and Thoracic Vascular Surgery, University Hospital Schleswig-Holstein, Lübeck, Germany, ${ }^{4}$ McKusick-Nathans Institute of Genetic Medicine, Johns Hopkins University School of Medicine, Baltimore, MD, United States, ${ }^{5}$ Department of Clinical Pathology, Lviv National Medical University after Danylo Halytsky, Lviv, Ukraine, ${ }^{6}$ Cardiovascular Research, SickKids University Hospital, Toronto, ON, Canada, ${ }^{7}$ Cardiovascular Medicine Unit, Department of Medicine, Karolinska Institute, Stockholm, Sweden, ${ }^{8}$ Cardiothoracic Surgery Unit, Department of Molecular Medicine and Surgery, Karolinska Institute, Stockholm, Sweden, ${ }^{9}$ Department of Clinical Genetics, Erasmus University Medical Center, Rotterdam, Netherlands, ${ }^{10}$ Assistance Publique-Hôpitaux de Paris, Hôpital Européen Georges Pompidou; Université Paris Descartes, Paris Sorbonne Cité; Institut National de la Santé et de la Recherche Médicale, UMRS, Paris, France, ${ }^{11}$ Department of Biology and Medical Genetics, 2nd Faculty of Medicine-Charles University and Motol University Hospital, Prague, Czechia, ${ }^{12}$ Institute of Clinical and Experimental Medicine, Prague, Czechia, ${ }^{13}$ Department of Human Genetics, Radboud University Medical Centre, Nijmegen, Netherlands, ${ }^{14}$ Howard Hughes Medical Institute, Baltimore, MD, United States

Keywords: bicuspid aortic valve, thoracic aortic aneurysm, SMAD6, targeted gene panel, variant burden test

\section{A corrigendum on}

Candidate Gene Resequencing in a Large Bicuspid Aortic Valve-Associated Thoracic Aortic Aneurysm Cohort: SMAD6 as an Important Contributor

by Gillis, E., Kumar, A. A., Luyckx, I., Preuss, C., Cannaerts, E., van de Beek, G., et al. (2017). Front. Physiol. 8:400. doi: 10.3389/fphys.2017.00400

In the original article, we noted two mutation annotation errors. The correction of these two mistakes does not change the scientific conclusions in any way. The authors apologize for these nomenclature errors. Please find below the corrected annotations of those two mutations:

(1) The correct RNA and protein annotations of the SMAD6 variant in P99 are c.455_461del and p.Pro152Profs*27, and not c.454_461del and p.Gly166Valfs*23.

(2) The correct RNA and protein annotations of the SMAD6 variant in P128 are c.74_79del and p.Ser27_Gly28del, and not c.73_79del and p.Gly26_Ser27del.

As a consequence, a correction has been made to RESULTS, Paragraphs 5 and 6 : 


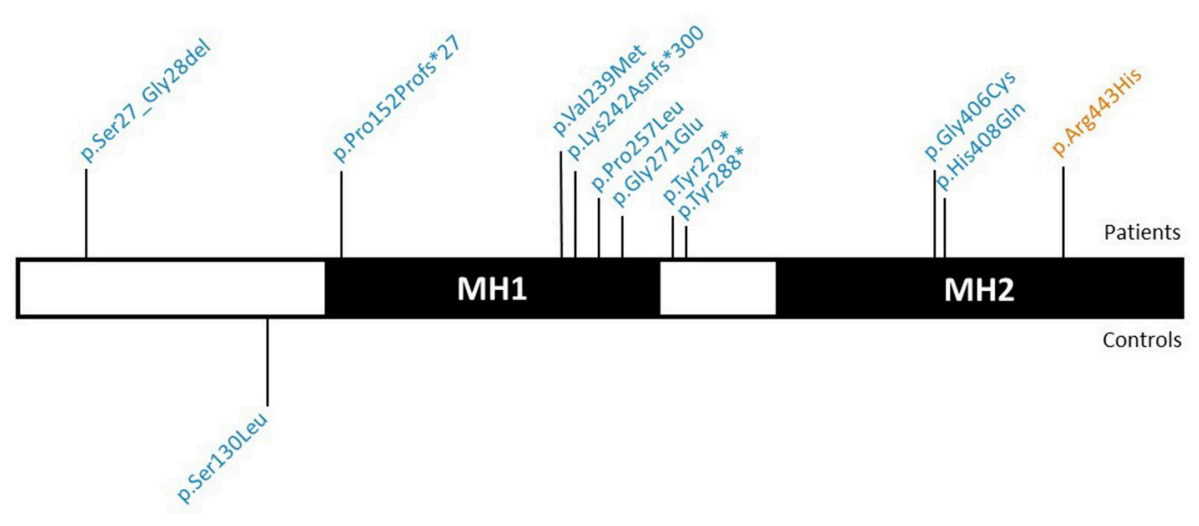

FIGURE 2 | Graphical representation of the identified SMAD6 variants. SMAD6 has two major protein domains, a DNA-binding MH1 domain and a MH2 domain that interacts with components of the TGF- $\beta$ and BMP signaling pathways. Variants above the protein have been found in patients, while those below the protein occurred in control individuals. Variants in blue are absent from the EXAC database, variants in orange have an EXAC MAF below $0.01 \%$. TGF- $\beta$, Transforming growth factor- $\beta$; BMP, Bone morphogenetic protein; ExAC, Exome Aggregation Consortium; MAF, Minor Allele frequency.

The SMAD6 c.726del variant leads to a frameshift (p.Lys242Asnfs*300) and a predicted protein with a Cterminal extension due to loss of the intended stop codon. The c.455_461del frameshift variant (p.Pro152Profs*27) causes the introduction of a premature stop codon, most likely resulting in haploinsufficiency due to nonsense-mediated mRNA decay (NMD). Also the two nonsense variants (p.Tyr279* and p.Tyr288*) are predicted to lead to NMD. All of the missense variants cluster in the functionally important $\mathrm{MH} 1$ and $\mathrm{MH} 2$ domains (Makkar et al., 2009) (amino acids 148-275 and 331-496, respectively), which is not the case for the sole missense variant (p.Ser130Leu) found in a control individual (Figure 2). All but one (p.Arg443His) of the identified variants were absent in the ExAC control cohort (v0.3.1; Supplementary Table 2). Moreover, the missense variants in the patient cohort (7/7) are enriched in the $\mathrm{MH} 1$ and $\mathrm{MH} 2$ domains when compared to ExAC controls $(n=228 / 430 ; p=0.02)$.

For two SMAD6 mutation carriers (P89, p.Gly271Glu; P99, p.Pro152Profs*27), gDNA of family members was available for

\section{REFERENCES}

Makkar, P., Metpally, R. P., Sangadala, S., and Reddy, B. V. (2009). Modeling and analysis of $\mathrm{MH} 1$ domain of Smads and their interaction with promoter DNA sequence motif. J. Mol. Graph. Model. 27, 803-812. doi: 10.1016/j.jmgm.2008. 12.003

Conflict of Interest Statement: The authors declare that the research was conducted in the absence of any commercial or financial relationships that could be construed as a potential conflict of interest. segregation analysis (Supplementary Figure 1). Although neither of these probands had a documented family history of BAV/TAA, a brother of P89 has been diagnosed with a sinus of Valsalva aneurysm $(45 \mathrm{~mm})$ and carried the SMAD6 mutation. The mutation was also observed in an unaffected daughter (age 28) of the proband (Supplementary Figure 1). Three unaffected siblings at ages 54, 58, and 64 did not carry the mutation. No gDNA was available from a sister of $\mathrm{P} 99$ with unspecified aortic valve problems. The p.Pro152Profs*27 mutation was found in an unaffected daughter (age 39) of P99 but was absent in his 37 year-old unaffected son (Supplementary Figure 1).

We also provide a corrected Figure 2 and Supplementary Table 2 .

\section{SUPPLEMENTARY MATERIAL}

The Supplementary Material for this article can be found online at: http://journal.frontiersin.org/article/10.3389/fphys. 2017.00730/full\#supplementary-material

Copyright (C) 2017 Gillis, Kumar, Luyckx, Preuss, Cannaerts, van de Beek, Wieschendorf, Alaerts, Bolar, Vandeweyer, Meester, Wünnemann, Gould, Zhurayev, Zerbino, Mohamed, Mital, Mertens, Björck, Franco-Cereceda, McCallion, Van Laer, Verhagen, van de Laar, Wessels, Messas, Goudot, Nemcikova, Krebsova, Kempers, Salemink, Duijnhouwer, Jeunemaitre, Albuisson, Eriksson, Andelfinger, Dietz, Verstraeten, Loeys and Mibava Leducq Consortium. This is an open-access article distributed under the terms of the Creative Commons Attribution License (CC $B Y$ ). The use, distribution or reproduction in other forums is permitted, provided the original author(s) or licensor are credited and that the original publication in this journal is cited, in accordance with accepted academic practice. No use, distribution or reproduction is permitted which does not comply with these terms. 